\title{
Propagation of Tremors over the Surface of an Elastic Solid.
}

\author{
By \\ Hitoshi TakeuchI \\ Geophysical Institute, Faculty of Science, Tolyo University, Tokyo. \\ and \\ Naota Kobayashi. \\ Departmert of Precision Mechanics, Faculty of Technology, Chüo University, Tolyo.
}

\begin{abstract}
Wave phenomena which are seen along the surface of a semi-infinite elastic solid when subjected to an impulsive line force in the direction normal to its surface have been studied. The results obtained are as follows.

(1) Immediately after the impulsive force is removed, the vertical displacement is downward in the neighbourhood of the origin, and it is upward outside that downward domain. (\$3)

(2) In the downward (upward) domain, the horizontal displacement is toward (away from) the origin. (\$ 4 )

(3) The surface deformations thus produced are propagated with the velocity of RAYLGIGH wave without conspicuous changes in form. (\$3 and \$4)

(4) The wave length of the RAYLEIGH wave is proportional to the time of duration of the impulse, and is of the same order of extent as that of the downward domain at the moment when the force is removed. (\$3)
\end{abstract}

$\S 1$. Wave phenomena which are seen along the surface of a semi-infinite elastic solid when subjected to an external impulsive force were studied by H. LAMB (1904). His results are chiefly concerned with the phenomena which are to be observed at the places far distant from the wave origin. The deformation of the elastic solid in the neighbourhood of the origin was not studied in his paper. On the other hand, an experimental approach to this problem has recently been made by K. Kasahara $(1952,1953)$. In his experiments, an apparently curious phenomenon has been found in the neighbourhood of the origin. He has stated: "When an impulsive vertical force acts downwards on the surface of an elastic medium, a part of the surface within a certain region begins in upward motion. The disturbance which occurs in such a way is propagated as time passes on. Such a phenomenon seems rather curious from the stand point of the theory of the statical deformation of elastic bodies. Because, as
Boussines? has studied, every part of the surface of a semi-infinite elastic body subjected to an external vertical force acting downwards at a point, is displaced downwards and no part showing upward displacements can exist on the surface. In fact, a statical experiment carried out on the same medium produces such results". Kasaefara has shown theoretically the possibility of swelling up of the surface of an elastic solid when subjected to an impulsive downward force, but has failed to bring a unified view in connecting this upward motion with the phenomena observed at the places far distant from the origin (1953). It is the purpose of the present paper to show a simple way of calculating the surface displacement of the elastic solid at any time and at any point and to fill up the gap between the knowledges concerning two extremes above cited.

$\S 2$. It was shown by LAMB (1904, his equation (93)) that the vertically downward displacement $v_{0}$ at $(x, y=0, t)$ due to the 
vertically downward force $Q(t)$ at the origin $(x=0, y=0)$ is given as follows.

$$
\begin{aligned}
v_{0}= & -\frac{1}{\pi \mu} \int_{a}^{b} \frac{b^{2}\left(2 \theta^{2}-b^{2}\right)^{2} V \theta^{2}-a^{2}}{\left(2 \theta^{2}-b^{2}\right)^{1}+16 \theta^{4}\left(\theta^{2}-a^{2}\right)\left(b^{2}-\theta^{2}\right)} Q(t-\theta x) d \theta \\
& -\frac{1}{\pi \mu} p \int_{b}^{\infty} \frac{b^{2} \sqrt{ } \theta^{2}-a^{2}}{\left(2 \theta^{2}-b^{2}\right)^{2}-4 \theta^{2} \sqrt{ } \theta^{2}-a^{2} \sqrt{\theta^{2}-b^{2}} Q(t-\theta x) d \theta .}
\end{aligned}
$$

In (2.1), $x$ is the distance of the point from the origin and $t$ is the time, $a$ and $b$ in (2.1) are

$$
a=\frac{1}{V_{p}}=\sqrt{\frac{\rho}{\lambda+2 \mu}}, \quad b=\frac{1}{V_{S}}=\sqrt{\frac{\rho}{\mu}}
$$

respectively and the symbol $p$ denotes the principal part of the integral which follows it. It is to be noted that the equation (2.1) is valid for any $x$ and $t$. Equation (2.1) can be rewritten as follows.

$$
\begin{gathered}
v_{0} \frac{V_{P}}{V_{S}} \pi \mu=p \int_{-\infty}^{+\infty} V\left(\frac{t-t_{0}}{a x}\right) Q\left(t_{0}\right) d\left(\frac{t-t_{0}}{a x}\right) \\
\quad=p \int_{-\infty}^{\infty} V\left(\begin{array}{c}
V_{P}\left(t-t_{0}\right) \\
x
\end{array}\right) Q\left(t_{0}\right) d\left(\begin{array}{c}
V_{P}\left(t-t_{0}\right) \\
x
\end{array}\right),
\end{gathered}
$$

in which $V\left(\theta^{\prime}\right)=V(\theta / a)$ is

$$
\begin{aligned}
& V\left(\theta^{\prime}\right)=-\left(\begin{array}{c}
m^{3}\left(2 \theta^{\prime 2}-m^{2}\right) \sqrt{\theta^{\prime 2}-1} \\
\left(2 \theta^{\prime 2}-m^{2}\right)^{4}+16 \theta^{\prime 4}\left(\theta^{\prime 2}-1\right)\left(m^{2}-\theta^{\prime 2}\right)
\end{array}\right. \\
& \text { for } \quad 1 \leq \theta^{\prime} \leq m \\
& V\left(\theta^{\prime}\right)=-\frac{m^{3} V \theta^{\prime 2}-1}{\left(2 \theta^{\prime 2}-m^{2}\right)^{2}-4 \theta^{\prime 2} \sqrt{\theta^{\prime 2}}-1 \sqrt{ } \theta^{\prime 2}-m^{2}} \\
& \text { for } m \leq \theta^{\prime} \\
& m=\frac{b}{a}=\frac{V_{P}}{V_{s}} .
\end{aligned}
$$

The value of $V\left(\theta^{\prime}\right)$ for the case $\lambda=\mu$ is given in the LAMB's paper. We reproduce it in Fig. 1.

We shall now calculate the value of $v_{0}$ in the case when

$$
Q\left(t_{0}\right)=\text { const } Q \text {, for }-\tau \leq t_{0} \leq \tau
$$

and $Q\left(t_{0}\right)=0$, otherwise.

Putting $\quad t=\tau t^{\prime} \quad t_{0}=\tau t_{0}{ }^{\prime}$

in (2.4), we have

$$
-\frac{m \pi \mu}{Q} v_{0}=p \int_{\frac{V_{p} \tau}{x}\left(t^{\prime}-1\right)}^{\frac{V_{p} \tau}{x}\left(t^{\prime}+1\right)}\left(-V\left(\theta^{\prime}\right)\right) d \theta^{\prime}
$$

This is the formula by which the value of

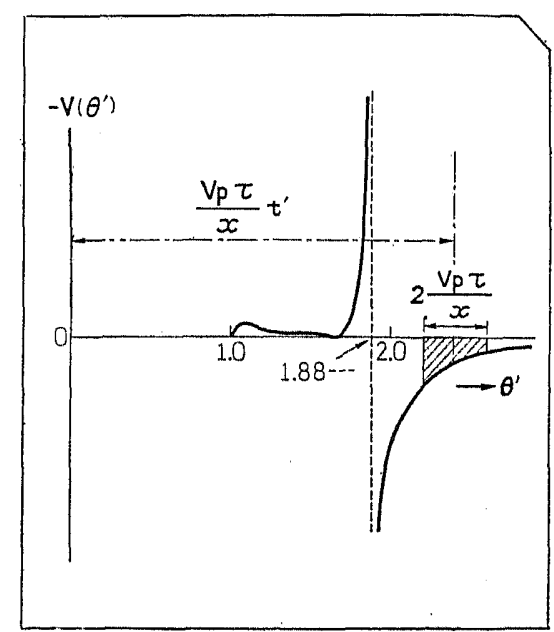

Fig. 1. The function $V\left(\theta^{\prime}\right)$

$v_{0}$ at any $x$ and $t$ can be calculated. The hatched area which is shown in Fig. 1 represents the integral on the right hand side of (2.7) corresponding to a certain $t^{\prime}$. In executing this integration for various values of $t^{\prime}$, we come across a difficulty when the domain of the integral $\int\left(-V\left(\theta^{\prime}\right)\right) d \theta^{\prime}$ includes $\theta^{\prime}=1.88 \ldots$ At $\theta^{\prime}=1.88 \ldots$ the integrand $-V\left(\theta^{\prime}\right)$ in (2.7) becomes infinite of the order of $\left(\theta^{\prime}-1.88 \ldots\right)^{-1}$. This difficulty is similar to that encounterd in executing the integral $\int_{-1}^{+1} d x$, for instance. The limiting process by the aid of the principal value expressed the symbol $p$ in (2.7) removes the difficulty stated above. Thus making use of (2.7), we can calculate the value of $-v_{0}$ (upward displacement) for any $x$ and $t$. The results of calculation for the case when $\lambda=\mu$ are shown in Fig. 2.

§3. As is seen in Fig. 2, the vertical displacement at $t=0$ or $t=\tau$ is downward for $\frac{x}{\tau V_{1}} \leq 0.49$ or $\frac{x}{\tau V_{1}} \leq 0.98$ respectively. It is of 


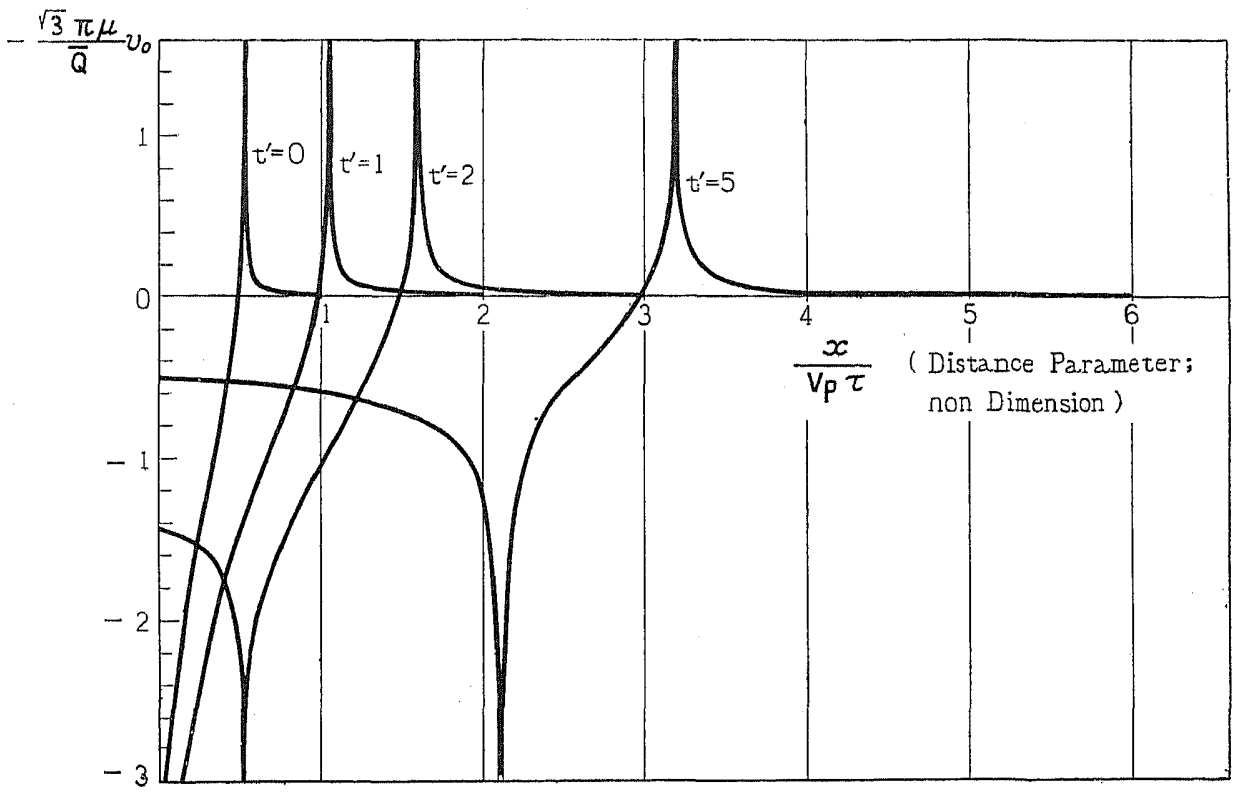

Fig. 2. Upward deformation $-v_{0}$

interest to note that the linear extension of the downward domain is proportional to $\tau$ (half the time of duration of the downward force). Thus if we make $\tau \rightarrow \infty$, we have the downward displacement everywhere on the surface of the elastic solid. This is nothing but the result obtained by Boussinese in the case of a statical downward force. There is a domain of the upward displacement outside the downward domain above stated. In the two dimensional problem as ours, the points of zero displacements lie on a straight line $n=$ const. at a fixed time. As time passes on, this line of zero displacement, i.e., the boundary line between the upward and the downward domains is propagated from the origin with the velocity of RAYLeigh wave. On the other hand, the front of the upward displacement is propagated from the origin with the velocity of $P$ wave (compressional wave). The origin times of these two "waves" are both $t=-\tau$. Thus the upward (and also of the downward) domain increases in its extent according to time. The peak of the upward displacement is propagated from the origin with the velocity of RAYLEIGH wave. The origin time of this upward peak is also $t=-\tau$, i.e., at the time when the vertical force begins to act. The trough of the downward displacement is propagated from the origin also with the velocity of RAYLEIGH wave. The origin time of this trough is $t=+\tau$, i.e., at the time when the vertical force is removed. The time interval at any point between the arrival times of the upward peak and the downward trough is $2 \pi$. The corresponding "wave length" $L$ of the RaYLwirar wave is $V_{l} \cdot 4 \tau$, that is, the RAYLEIGH wave velocity times twice the time of duration of the downward force. As was stated at the beginning of the present section, the domain of the downward displacement at $t=\tau$ is characterised by the relation $|x| \leq 0.98 \tau V_{l}$. and the width $D$ of the domain of the downward displacement is $2 \times 0.98 \tau V_{p^{\prime}}$. Making use of the relation $V_{R}=0.53 V_{R}$ for the case when $\lambda=\mu$, we have $D=3.7 \tau V_{\mu}$. This is approximately equal to the wave length $L=4 \tau V_{R}$ of the generated RAYLisigh wave.

In Fig. 2 are shown only the displacements up to the time $t=5 \tau$. The approximate pattern of the displacement for $t^{\prime}>5$, however, can be obtained as follows. The front of the upward displacement is at $x=V_{P^{\prime}}(t+\tau)$. The 
peak of the upward displacement and the trough of the downward displacement are at $x=V_{R}(t+\tau)$ and $V_{R}(t-\tau)$ respectively. The displacement in the neighbourhood of the origin decreases gradually as time goes on. In other respect, the pattern of the displacement is similar to that at $t \geq 5 \tau$.

The curve of displacement at $x=0$ is shown in Fig. 3.

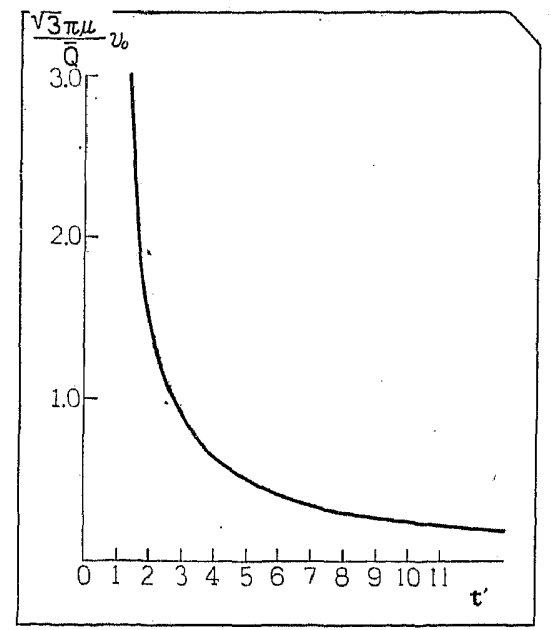

Fig. 3. Displacement at the origin

For the large values of $t=\tau t^{\prime}$, the curve is expressed approximately by the equation

$$
-\frac{V \pi \mu}{Q} v_{0}=\frac{3 V 3}{4} \log _{0}\left(1+\frac{2}{t^{\prime}}\right) .
$$

The equation (3.1) is obtained as follows. From (2.4), we have

$$
-V\left(\theta^{\prime}\right)=\frac{3 \sqrt{ } 3}{4} \theta^{\prime-1}
$$

for the large $\theta^{\prime}$. Inserting (3.2) into (2.7), we get the equation (3.1). It can readily be seen that all of the numerical results above obtained are closely connected to the infinity point at $\theta^{\prime}=1.88 \ldots$ of the function $-V\left(\theta^{\prime}\right)$ in Fig. 1. This infinity point at $\theta^{\prime}=1.88 \ldots$ is, in turn, closely connected to the RAYLEIGH wave velocity for the case when $\lambda=\mu$. In fact, the numerical factor 0.53 in the relation $V_{R}=0.53 V_{p}$ is nothing but the reciprocal of this $1.88 \ldots$ It is therefore expected that the similar results as those obtained above will be obtained for the elastic solid for which $\lambda \neq \mu$. KaSAHARA's experiment referred to at the beginning of the present paper is made with an elastic solid (agar-agar) for which $\lambda=4 \sim 5 \mu$. Adopting this value of $\lambda / \mu$, his experimental results agree quite well with the above theoretical results. According to KASAHARA, for example, the peak of the upward deformation in his experiment is propagated with the velocity of about $1 \mathrm{~cm} / 5 \mathrm{~m} \mathrm{sec}$ $=200 \mathrm{~cm} / \mathrm{sec}$. (see Fig. 4 in his paper II) and this is seen from Table II in the same paper of his to be about the velocity of the RAYLErGH wave in his experiment, just as is expected from our calculations.

$\S 4$. By the similar methods as adopted in the preceding sections, we can calculate the horizontal displacement $u_{0}(x, y=0, t)$. The equation by which the value of $u_{0}$ is to be calculated is as follows

$$
\begin{aligned}
\frac{m \pi \mu}{Q} u_{0}=-m \pi H \delta & \left(t^{\prime}-t_{0}-\begin{array}{c}
x \\
V_{p \tau} \tau
\end{array}\right) \\
& +2 \int_{\frac{V_{p} \tau}{x}\left(t^{\prime}-1\right)}^{\frac{V_{p} \tau}{x}\left(t^{\prime}+1\right)} U\left(\theta^{\prime}\right) d \theta^{\prime}
\end{aligned}
$$

in which $\delta$ is the Drrac's function, and

$$
\begin{gathered}
U\left(\theta^{\prime}\right)=-\frac{m^{3} \theta^{\prime}\left(2 \theta^{\prime 2}-m^{2}\right) \sqrt{\theta^{\prime 2}-1 \sqrt{ } m^{2}-\theta^{\prime 2}}}{\left(2 \theta^{\prime 2}-m^{2}\right)^{1}+16 \theta^{\prime 4}\left(\theta^{\prime 2}-1\right)\left(m^{2}-\theta^{\prime 2}\right)} \\
1 \leq \theta^{\prime} \leq m \\
U\left(\theta^{\prime}\right)=0, \quad \theta^{\prime} \leq 1, \quad \theta^{\prime}>m \\
H=0.12500 \quad(\lambda=\mu) .
\end{gathered}
$$

The equation (4.1) and (4.2) are derived from the equation (92) in the LAMB's paper, and the value of $U\left(\theta^{\prime}\right)$ for the case when $\lambda=\mu$ was calculated also in the LAMB's paper. Thus making use of (4.1), we can calculate the value of $u_{0}$ for any $x$ and $t$. The results of calculation for the case when $\lambda=\mu$ are shown in Fig. 4.

As is seen from Fig. 4, the horizontal displacement at $t=0$ or $t=\tau$ is towards the origin for $x$ less than about $0.57 \tau V_{P}$ or $1.15 \tau V_{P}$, respectively. This domain of inward displacement roughly corresponds to the downward domain referred to at the beginning of the last section. Outside this domain, there is also a 


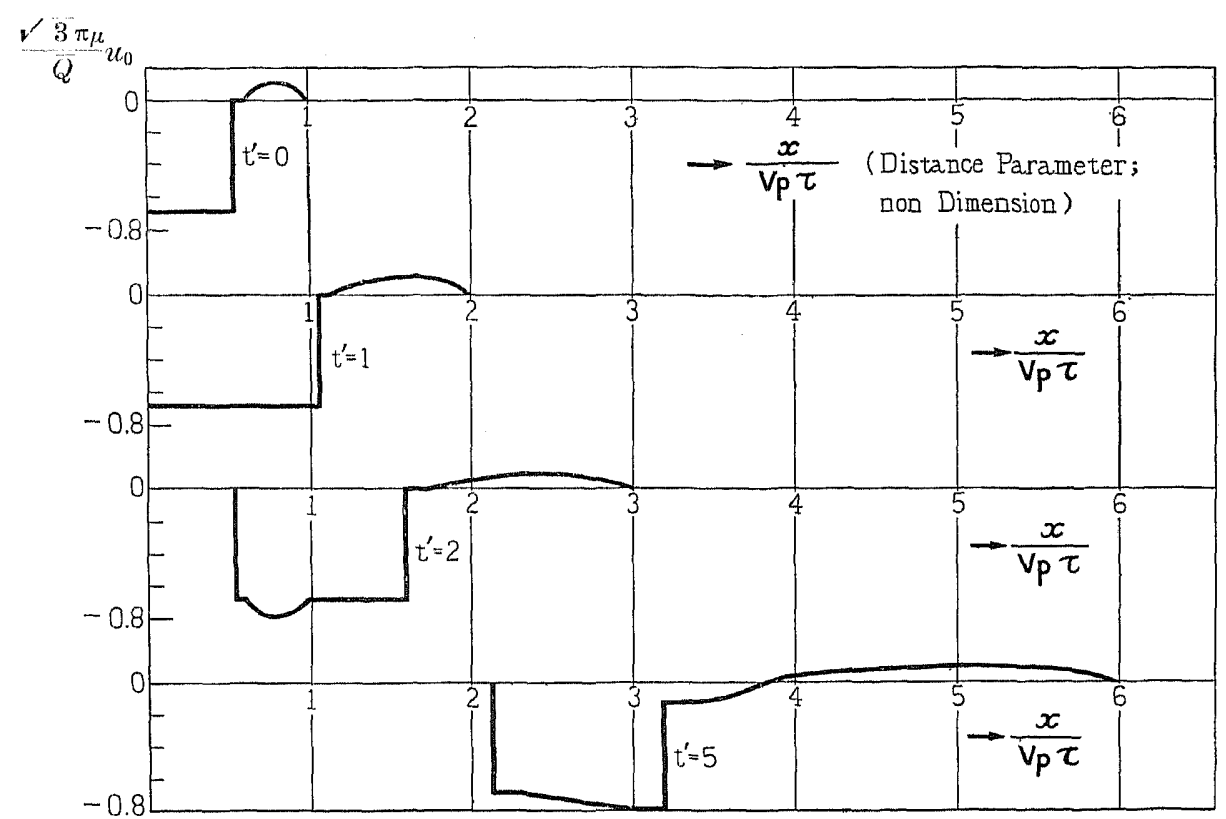

Fig. 4. Horizontal Displacement $u_{0}$

domain in which the horizontal displacement is outward from the origin. In the "outward domain", the vertical deformation is upward. The front of this horizontal displacement is propagated with the velocity of $P$ wave. The origin time of this wave is $t=-\tau$. The principal part of the horizontal displacement at $t$ is limited in the interval

$$
V_{l i}(t-\tau) \leq x \leq V_{k}(t+\tau) .
$$

The value of $u_{0}$ in this part is about $-0.7 \frac{6}{\sqrt{3} \pi / 2}$. The front and rear of the principal part are propagated from the origin with the velocity of Raylergra wave. The origin time of these front and rear waves are $t=-\tau$ and $t=\tau$ respectively.

$\S 5$. Throughout the present study, a line source of wave has been assumed, and the problem has been treated as a two-dimensional one. In the two-dimensional problem as ours, it is to be noted that the waves are propagated without conspicuous changes in form. In a three-dimensional problem with a point source of force, this is not the case. This latter problem will be studied in another paper.

\section{References}

Kasahara, K.:

1952 "Experimental studies on the mechanism of generation of elastic waves 1". Bull. Earthq. Res. Inst., 30, 259.

1953 "Experimental studies on the mechanism of generation of elastic waves $2 "$. Bull. Earthq. Res. Inst., 31, 71.

LAMB, H.:

1904 "On the propagation of tremors over the surface of an elastic solid" Phil. Trans., A 203, 1. 